\title{
OSCILLATION CRITERIA AND GROWTH OF NONOSCILLATORY SOLUTIONS OF EVEN ORDER ORDINARY AND DELAY-DIFFERENTIAL EQUATIONS
}

\author{
BY \\ R. GRIMMER
}

\begin{abstract}
A number of results are presented on oscillation and growth of nonoscillatory solutions of the differential equation $x^{(n)}(t)+f(t, x(t))=0$. It is shown that a nonoscillatory solution satisfies a first-order integral inequality while its $(n-1)$ st derivative satisfies a first-order differential inequality. By applying the comparison principle, results are obtained by analyzing the two associated first-order scalar differential equations. In the last section it is shown that these results can be easily extended to delay-differential equations.
\end{abstract}

1. Introduction. In this paper we examine the differential equation

$$
x^{(n)}+f(t, x)=0 \quad(n \text { even })
$$

where $f(t, x)$ is continuous for all real $x$ and $t \geq 0$, satisfies $x f(t, x)>0$ for $x \neq 0$, and $f(t, x)$ is nondecreasing in $x$ for fixed $t$.

For the purposes of this paper we define a solution of (1.1) to be oscillatory if it has no last zero and nonoscillatory if it either has no zeros or a last zero. As every solution $x(t)$ of (1.1) satisfies $x(t) x^{(n)}(t)<0$ for those values of $t$ for which $x(t) \neq 0$, it is clear that a nonoscillatory solution of (1.1) can be extended to a positive half-line. However, an oscillatory solution may have finite escape time ([3], [8]), depending on the smoothness of $f$. For the purposes of this paper it will not be convenient to rule out such behavior.

The problem of determining if all solutions of equation (1.1) are oscillatory has been the subject of an extensive amount of study during the past several years. For results on oscillation of solutions of ordinary differential equations we cite the results of Kartsatos [9], Kiguradze [10], [11], Ličko and Švec [14], Onose [17], and Ryder and Wend [18] as being representative.

A well-known result on oscillation was obtained by Ličko and Švec [14] for the ordinary differential equation

$$
x^{(n)}+p(t) x^{\gamma}=0 \quad(n \text { even })
$$

where $p(t)>0$ is continuous on $[0, \infty)$ and $\gamma$ is the quotient of positive odd integers. Their result is the following:

Presented to the Society, January 26, 1973 under the title Oscillation and nonoscillation of even order differential equations, received by the editors January 15,1973 and, in revised form, April 23, 1973.

AMS (MOS) subject classifications (1970). Primary 34C10, 34K15.

Key words and phrases. Oscillation, growth, comparison method. 
Theorem A. If $\gamma>1$, every solution of (1.2) is oscillatory if and only if

$$
\int^{\infty} t^{n-1} p(t) d t=\infty
$$

If $0<\gamma<1$, every solution of (1.2) is oscillatory if and only if

$$
\int^{\infty} t^{(n-1)} p(t) d t=\infty
$$

We observe that Theorem A may be stated in a different manner, relating the oscillatory behavior of the solutions of (1.2) to the behavior of the solutions of the first-order equations

$$
u^{\prime}=t^{n-1} p(t) u^{\gamma}
$$

and

$$
v^{\prime}=-t^{\gamma^{(n-1)}} p(t) v^{\gamma}
$$

in the following way.

Theorem B. If $\gamma>1$, every solution of (1.2) is oscillatory if and only if every nontrivial solution of (1.5) has finite escape time. If $0<\gamma<1$, every solution of (1.2) is oscillatory if and only if every solution of (1.6) becomes zero in finite time.

An obvious proof of Theorem B is to show that conditions (1.3) and (1.4) are the necessary and sufficient conditions for the desired properties of the solutions of (1.5) and (1.6) respectively. However, there is a more fundamental relationship between equation (1.2) and equations (1.5) and (1.6). It will be shown that if $x(t)$ is a positive nonoscillatory solution of $(1.1)$ and $t_{1}$ is sufficiently large, $x(t)$ satisfies $x(t) \geq u\left(t ; t_{1}, x\left(t_{1}\right)\right)$ and $x^{(n-1)}(t) \leq v\left(t ; t_{1}, x^{(n-1)}\left(t_{1}\right)\right)$ for $t \geq t_{1}$, where $u\left(t ; t_{1}\right.$, $\left.x\left(t_{1}\right)\right)$ is a solution of an equation similar to (1.5) and $v\left(t ; t_{1}, x^{(n-1)}\left(t_{1}\right)\right)$ is a solution of an equation similar to (1.6). From these estimates explicit estimates on all nonoscillatory solutions of (1.1) will be obtained and, also, general conditions for the oscillation of all solutions of (1.1).

In the last section we shall see that these results extend in a natural way to the delay-differential equation

$$
x^{(n)}(t)+h\left(t, x(t), x\left(q_{1}(t)\right), \ldots, x\left(q_{m}(t)\right)\right)=0 .
$$

For results on oscillation of solutions of delay-differential equations we refer the reader to Burton and Grimmer [1], [2], Erbe [4], Gollwitzer [5], and Wong [19] for the second-order case and Ladas [12] for the higher order case.

2. Main results. Our results on the behavior of the solutions of (1.1) will involve the first-order scalar differential equations

$$
u^{\prime}=(t-a)^{n-1} f(t, u) /(n-1) !
$$


and

$$
v^{\prime}=-f\left(t, d t^{n-1} v\right)
$$

where $d=\left(\frac{1}{2}\right)^{n(n-1)}(1 /(n-1) !)$. Throughout this paper $u\left(t ; a, u_{0}\right)$ will denote the minimal (maximal) solution of (2.1a) with $u(a)=u_{0}$ if $u_{0}>0\left(u_{0}<0\right)$. In a similar fashion, $v\left(t ; a, v_{0}\right)$ will denote the maximal (minimal) solution of $(2.2)$ with $v(a)=v_{0}$ if $v_{0}>0\left(v_{0}<0\right)$.

We shall need the following basic lemma due to Kiguradze (cf. also [12], [18]).

Lemma 1 ([10], [11]). If $y(t)$ is a function such that it and all its derivatives up to order $(m-1)$ inclusive are absolutely continuous and of constant sign in the interval $\left(t_{0}, \infty\right)$, and $y^{(m)}(t) y(t) \leq 0$ for $t \geq t_{0}$, then there is an integer $l, 0 \leq l<m$, which is odd if $m$ is even and even if $m$ is odd, so that for $t \geq t_{0}$ we have the following:

(i) $y^{(k)}(t) y(t) \geq 0, k=0,1, \ldots, l$;

(ii) $(-1)^{m+k-1} y^{(k)}(t) y(t) \geq 0, k=l+1, \ldots, m$;

(iii) $|y(t)| \geq M\left(t-t_{0}\right)^{m-1}\left|y^{(m-1)}(N t)\right|$ where $M=(m-1-l) ! /(m-1)$ ! and $N=2^{m-l-1}$

(iv) $\left(t-t_{0}\right)\left|y^{(l-k)}(t)\right| \leq(1+k)\left|y^{(l-k-1)}(t)\right|, k=1, \ldots, l-1$;

(v) $|y(t)| \geq\left(t-t_{0}\right)^{\prime}\left|y^{(t)}(t)\right| / t$.

Theorem 1. If $x(t)$ is a nonoscillatory solution of (1.1), there exists $t_{0} \geq 0$ so that, for every $t_{1} \geq t_{0},|x(t)| \geq\left|u\left(t ; t_{1}, x\left(t_{1}\right)\right)\right|$ for $t \geq t_{1}$. Also, there exists $t_{2} \geq 0$ with the property that $\left|x^{(n-1)}(t)\right| \leq\left|v\left(t ; t_{3}, x^{(n-1)}\left(t_{3}\right)\right)\right|$ for $t \geq t_{3}$ for each $t_{3} \geq t_{2}$.

Proof. Let $x(t)$ be a nonoscillatory solution of (1.1). We shall assume that $x(t)$ is positive for all large values of $t$, the case when $x(t)$ is eventually negative is similar and will be omitted.

If $x(t)$ is positive for $t \geq T$, we see that, also, $x^{(n)}(t)<0$ for $t \geq T$. Furthermore, we must have $x^{(n-1)}(t)>0$ for $t \geq T$ as two consecutive negative derivatives of $x(t)$ would imply $x(t)$ has a zero at some $t>T$. In a similar fashion it is easy to see that there exists $t_{0} \geq T$ so that $x^{(j)}(t)$ is of constant sign for $t \geq t_{0}$ for $j=0,1, \ldots, n$.

If we multiply equation (1.1) by $\left(t-t_{1}\right)^{n-1}$ where $t_{1} \geq t_{0}$, an integration leads to the equation

$$
\begin{aligned}
x(t)= & x\left(t_{1}\right)+\sum_{j=1}^{n-1}(-1)^{j+1}\left(t-t_{1}\right)^{j} x^{(j)}(t) / j ! \\
& +\int_{t_{1}}^{t}\left[\left(s-t_{1}\right)^{n-1} f(s, x(s)) /(n-1) !\right] d s .
\end{aligned}
$$

It follows now from Lemma 1(i) and (ii) that there is an odd integer $l$ so that $x^{(j)}(t)>0$ for $j=1, \ldots, l$, and $(-1)^{j+1} x^{(j)}(t)>0$ for $j=l+1, \ldots, n$ for $t \geq t_{1}$ and, hence,

$$
\sum_{j=l}^{n-1}(-1)^{j+1}\left(t-t_{1}\right)^{j} x^{(j)}(t) / j ! \geq 0
$$


for $t \geq t_{1}$. Now, if $l>1$, defining $\psi(t)$ by

$$
\psi(t)=\sum_{j=1}^{l-1}(-1)^{j+1}\left(t-t_{1}\right)^{j} x^{(j)}(t) / j !
$$

we see that $\psi^{\prime}(t)=x^{\prime}(t)-\left(t-t_{1}\right)^{l-1} x^{(l)}(t) /(l-1)$ ! and it follows from an application of Lemma $1(\mathrm{v})$ to $y(t)=x^{\prime}(t)$ that $\psi^{\prime}(t) \geq 0$ for $t \geq t_{1}$. As $\psi\left(t_{1}\right)=0$, it follows from (2.3) that for $t \geq t_{1}, x(t)$ satisfies

$$
x(t) \geq x\left(t_{1}\right)+\int_{t_{1}}^{t}\left[\left(s-t_{1}\right)^{n-1} f(s, x(s)) /(n-1) !\right] d s .
$$

As $f(t, x)$ is nondecreasing in $x$, the first statement of the theorem now follows from a standard comparison theorem [13, p. 324].

To prove the second part of the theorem we define $t_{2}=2^{n} t_{0}$ and consider $x(t)$ for $t \geq t_{2}$. From Lemma 1(iii) and the fact that $x(t)$ is increasing we obtain

$$
\begin{aligned}
x(t) & \geq x\left(2^{1-n} t\right) \\
& \geq\left(2^{1-n} t-t_{0}\right)^{n-1} x^{(n-1)}(t) /(n-1) ! \\
& \geq d t^{n-1} x^{(n-1)}(t) .
\end{aligned}
$$

From (1.1) we see that, for $t \geq t_{2}, x(t)$ satisfies

$$
x^{(n)}(t) \leq-f\left(t, d t^{n-1} x^{(n-1)}(t)\right)
$$

Applying a standard comparison theorem for differential inequalities [13, p. 15] yields the desired result.

It is frequently the case that equation (2.1a) has a separatrix solution, that is, a solution $u^{*}(t)$ that exists on $[a, \infty)$ with the property that any solution $u(t)$ of (2.1a) with $u\left(t_{0}\right)>u^{*}\left(t_{0}\right)$ for some $t_{0} \geq a$ has finite escape time. This concept was examined extensively by Hallam and Heidel [7] and motivates our next theorem which frequently will enable one to obtain an explicit upper or lower bound for all nonoscillatory solutions of (1.1).

Theorem 2. Let $\Psi$ and $\theta$ be nonnegative functions defined for $a \geq 0$ with the property that $u_{0}>\Psi(a)$ implies $u\left(t ; a, u_{0}\right)$ has finite escape time and $v_{0}<\theta(a)$ implies there exists $t \geq a$ so that $v\left(t ; a, v_{0}\right) \leq 0$. If $x(t)$ is an eventually positive nonoscillatory solution of (1.1), there exists $t_{0} \geq 0$ so that $x(t) \leq \Psi(t)$ and $x^{(n-1)}(t) \geq \theta(t)$ for $t \geq t_{0}$. A similar statement also holds for negative nonoscillatory solutions.

Proof. If $x(t)$ is a positive nonoscillatory solution of (1.1) we have from Theorem 1 that there exists $t_{0} \geq 0$ with the property that, for every $t_{1} \geq t_{0}, x(t)$ $\geq u\left(t ; t_{1}, x\left(t_{1}\right)\right)$ and $x^{(n-1)}(t) \leq v\left(t ; t_{1}, x^{(n-1)}\left(t_{1}\right)\right)$ for $t \geq t_{1}$. Now if, for some $t_{1} \geq t_{0}, x\left(t_{1}\right)>\Psi\left(t_{1}\right), x(t)$ must have finite escape time which is a contradiction for, as we noted earlier, nonoscillatory solutions cannot have finite escape time. 
If, for some $t_{1} \geq t_{0}, x^{(n-1)}\left(t_{1}\right)<\theta\left(t_{1}\right)$, we must have $x^{(n-1)}\left(t_{2}\right) \leq 0$ for some $t_{2} \geq t_{1}$. This implies, however, that $x^{(n)}(t)$ and $x^{(n-1)}(t)$ are both negative after $t_{2}$ and so $x(t)$ must have a zero after $t_{1}$. This contradiction completes the proof.

Theorem 3. Every solution of (1.1) is oscillatory if any one of the following conditions are valid.

(i) For each $a>0$, every nontrivial solution of (2.1a) has finite escape time.

(ii) For each $a>0$ and $v_{0} \neq 0$ there exists $t>a$ so that $v\left(t ; a, v_{0}\right)=0$.

(iii) For each $a>0$ and $u_{0}>0, u\left(t ; a, \pm u_{0}\right)$ exists on $[a, \infty)$ and

$$
\int^{\infty} f\left(t, u\left(t ; a, \pm u_{0}\right)\right) d t= \pm \infty .
$$

Proof. The first two parts of the theorem follow immediately from Theorem 2.

Now suppose (iii) is valid and $x(t)$ is a nonoscillatory solution of (1.1). Assume $x(t)$ is positive for all large $t$ and choose $t_{0}>0$ having the property that $x\left(t_{0}\right)>0$ and $x(t) \geq u\left(t ; t_{0}, x\left(t_{0}\right)\right)$ for $t \geq t_{0}$. From (1.1) we obtain

$$
x^{(n-1)}(t)-x^{(n-1)}\left(t_{0}\right) \leq-\int_{t_{0}}^{t} f\left(s, u\left(s, t_{0}, x\left(t_{0}\right)\right)\right) d s
$$

and it follows that $x^{(n-1)}(t)$ must eventually become negative and $x(t)$ must, thus, have a zero after $t_{0}$, which is a contradiction. The case when $x(t)$ is eventually negative is essentially the same and is omitted.

3. Applications. The parts (i), (ii) and (iii) of Theorem 3 may be thought of as applying to equations which are essentially superlinear, sublinear, and linear, respectively. In this section we apply the results of Theorems 1-3 to these categories of equations.

We first examine the linear equation

$$
x^{(n)}(t)+p(t) x(t)=0 .
$$

Theorem 4. Suppose $p(t)$ is continuous and positive for $t>0$. If $x(t)$ is a nonoscillatory solution of (3.1),

$$
|x(t)| \geq|x(a)| \exp \int_{a}^{t}\left[(s-a)^{n-1} p(s) /(n-1) !\right] d s
$$

for some $a>0$. Every solution of (3.1) is oscillatory if, for each $a>0$,

$$
\int_{a}^{\infty} p(t) \exp \int_{a}^{t}\left[(s-a)^{n-1} p(s) /(n-1) !\right] d s d t=\infty .
$$

Proof. For equation (3.1), the corresponding first-order equation (2.la) takes the form

$$
u^{\prime}=(t-a)^{n-1} p(t) u /(n-1) !
$$

and the result follows from Theorem 1 and Theorem 3(iii). 
Before proceeding to nonlinear equations it will be necessary to examine the first-order scalar equations

$$
r^{\prime}=\omega_{1}(t, r)
$$

and

$$
r^{\prime}=-\omega_{2}(t, r)
$$

where, for $i=1,2, \omega_{i}(t, r)$ is continuous for all real $r$ and $t \geq 0$ and satisfies $\omega_{i}(t, r) r>0$ for $r \neq 0$.

We assume the existence of functions $k, l$, and $m$ which satisfy:

The functions $k, l$, and $m$ are continuous for all real $r, r k(r)>0, r l(r)>0, m(r)>0$ for $r \neq 0$ and $m\left(r_{1}\right) \leq m\left(r_{2}\right)$ if $0 \leq r_{1} \leq r_{2}$ or $r_{2} \leq r_{1} \leq 0$. In addition,

$$
\int_{ \pm 1}^{ \pm \infty} \frac{d r}{k(r)}=\infty, \quad \int_{ \pm 1}^{ \pm \infty} \frac{d r}{l(r)}<\infty
$$

There exist continuous nonnegative functions $b(t)$ and $c(t)$ defined for $t \geq 0$ so that $\omega_{1}(t, r) r \geq b(t) k(r) r$ and $\omega_{1}(t, r) r \geq c(t) l(r) m(r) r$.

We define functions $K_{i}(r)$ and $L_{i}(r), i=1,2$, for $(-1)^{i} r<0$ by

$$
K_{1}(r)=\int_{1}^{r} \frac{d u}{k(u)}, \quad K_{2}(r)=\int_{-1}^{r} \frac{d u}{k(u)}
$$

and

$$
L_{\cdot 1}(r)=\int_{1}^{r} \frac{d u}{l(u)}, \quad L_{2}(r)=\int_{-1}^{r} \frac{d u}{l(u)}
$$

Lemma 2. If (3.4) and (3.5) are valid, every nontrivial solution of (3.2) has finite escape time if, for each $p \neq 0$ and $a \geq 0$,

$$
\int_{a}^{\infty} c(t) m\left(K_{i}^{-1}\left(K_{i}(p)+\int_{a}^{t} b(s) d s\right)\right) d t=\infty
$$

where $(-1)^{i} p<0$ and $K_{i}^{-1}$ is the inverse of $K_{i}$.

Proof. Let $r(t)$ be a solution of (3.2) with initial condition $r(a)=p>0$. From (3.2) and (3.5), $r^{\prime}(t) \geq b(t) k(r)$ and, hence, for $t \geq a$,

$$
r(t) \geq K_{1}^{-1}\left(K_{1}(p)+\int_{a}^{t} b(s) d s\right) .
$$


Also, it follows from (3.5) that

$$
\begin{aligned}
r^{\prime}(t) & \geq c(t) l(r) m(r) \\
& \geq c(t) l(r) m\left(K_{1}^{-1}\left(K_{1}(p)+\int_{a}^{t} b(s) d s\right)\right)
\end{aligned}
$$

and as long as $r(t)$ exists, for $t \geq a$,

$$
L_{1}(r(t))-L_{1}(p) \geq \int_{a}^{t} c(u) m\left(K_{1}^{-1}\left(K_{1}(p)+\int_{a}^{u} b(s) d s\right)\right) d u .
$$

It now follows from (3.4) that $r(t)$ cannot exist on $[a, \infty)$. The argument when $p<0$ is similar and is omitted.

Lemma 3. Suppose (3.4) and (3.5) are valid and $\Psi>0$ is a constant with the property that

$$
L_{1}(\infty)-L_{1}(\Psi)<\int_{a}^{\infty} c(t) m\left(K_{1}^{-1}\left(K_{1}(\Psi)+\int_{a}^{t} b(s) d s\right)\right) d t .
$$

If $r(t)$ is a solution of (3.2) with $r(a)=p \geq \Psi$, then $r(t)$ cannot be continued to $[a, \infty)$. A similar statement is valid for negative solutions of (3.2).

Proof. Let $r(t)$ be a solution of (3.2) with $r(a)=p \geq \Psi$. If $r(t)$ exists for all $t \geq a$, we obtain, from (3.8),

$$
\begin{aligned}
L_{1}(\infty)-L_{1}(\Psi) & \geq L_{1}(r(\infty))-L_{1}(p) \\
& \geq \int_{a}^{\infty} c(u) m\left(K_{1}^{-1}\left(K_{1}(p)+\int_{a}^{u} b(s) d s\right)\right) d u \\
& \geq \int_{a}^{\infty} c(u) m\left(K_{1}^{-1}\left(K_{1}(\Psi)+\int_{a}^{u} b(s) d s\right)\right) d u
\end{aligned}
$$

as $K_{1}$ is an increasing function. However, from (3.9) we have the absurdity $L_{1}(\infty)-L_{1}(\Psi)>L_{1}(\infty)-L_{1}(\Psi)$, and the proof is complete.

To determine if all nontrivial solutions $r(t)$ of (3.3) become zero in finite positive time it suffices to consider $v(t)=1 / r(t)$. Clearly, $r(t)$ becomes zero in finite time if and only if $v(t)$ does not exist on a positive half-line. As $v(t)$ satisfies

$$
v^{\prime}=v^{2} \omega_{2}(t, 1 / v)=\omega_{3}(t, v),
$$

we may apply Lemmas 2 and 3 to equations (3.10) to obtain corresponding results for equation (3.3) if (3.4) and (3.5) are valid with $\omega_{1}(t, r)$ replaced by $\omega_{3}(t, r)$.

The method of analysis of equations (3.2) and (3.3) is similar to that used in [6] and [7] and Lemmas 2 and 3 are related to Theorems 4 and 1, respectively, of [7]. In certain cases, Lemma 2 will yield information while Theorem 4 of [7] does 
not. This can be seen by examining the equation

$$
r^{\prime}=b(t) r^{\beta}+c(t) r^{\gamma}
$$

where $b(t)$ and $c(t)$ are positive and continuous for $t \geq 0$ and $\beta$ and $\gamma$ are quotients of odd integers satisfying $0<\beta<1<\gamma$. The result of [7] requires that all nontrivial solutions of

$$
r^{\prime}=c(t) r^{\gamma}
$$

have finite escape time to guarantee the same property for equation (3.11) while Lemma 2 does not require this.

We now consider a class of equations which it is convenient to think of as being essentially superlinear.

Theorem 5. Suppose that $x f(t, x) \geq x[b(t) k(x)+c(t) l(x) m(x)]$ with $b$ and $c$ continuous and nonnegative for $t \geq 0, k, l$ and $m$ continuous for all $x$ and satisfying (3.4).

(i) Every solution of (1.1) is oscillatory if, for each $p \neq 0$ and $a \geq 0$,

$$
\int^{\infty} C(t, a) m\left(K_{i}^{-1}\left(K_{i}(p)+\int_{a}^{t} B(s, a) d s\right)\right) d t=\infty
$$

where $(-1)^{i} p<0$.

(ii) Suppose $\Psi_{1}(t)>0>\Psi_{2}(t)$ are functions which satisfy

$$
\begin{aligned}
L_{i}( \pm \infty)-L_{i}\left(\Psi_{i}(a)\right)<\int_{a}^{\infty} C(t, a) m\left(K_{i}^{-1}\left(K_{i}\left(\Psi_{i}(a)\right)+\int_{a}^{t} B(s, a) d s\right)\right) d t & \\
i & =1,2 .
\end{aligned}
$$

If $x(t)$ is a nonoscillatory solution of (1.1), for all large $t, x(t)$ satisfies $\Psi_{1}(t)>x(t)$ $>\Psi_{2}(t)$.

(iii) If $x(t)$ is a nonoscillatory solution of (1.1),

$$
L_{i}(x(t)) \geq L_{i}(x(a))+\int_{a}^{t} C(u, a) m\left(K_{i}^{-1}\left(K_{i}(x(a))+\int_{a}^{u} B(s, a) d s\right)\right) d u
$$

for $t \geq a$, a sufficiently large, where $(-1)^{i} x(t)<0$ for $t \geq a$.

Here, $B(t, a)=(t-a)^{n-1} b(t) /(n-1) !$ and $C(t, a)=(t-a)^{n-1} c(t) /(n-1)$ !.

Proof. The proof is immediate, (i) following from Lemma 2 and Theorem 3(i), (ii) from Lemma 3 and Theorem 2, while (iii) follows from Theorem 1 and (3.8).

By taking $m(x) \equiv 1$ and $b(t) \equiv 0$ we see that Theorem 5(i) includes Theorem 2 of [9] and the sufficiency criterion for oscillation for the superlinear equation given by Theorem A. While part (iii) of Theorem 5 has little content when $b(t) \equiv 0$ and $m(x) \equiv 1$, it is of interest to examine part (ii). 
Corollary 1. Suppose that $x f(t, x) \geq c(t) l(x) x$ where $c(t)$ is positive and continuous for $t \geq 0, l$ is continuous for all $x, x l(x)>0$ for $x \neq 0$ and $\int_{ \pm 1}^{ \pm \infty} d u / l(u)$ $<\infty$. If $x(t)$ is a nonoscillatory solution of (1.1), there exists $t_{0} \geq 0$ so that, for $t \geq t_{0}$

$$
|x(t)| \leq L_{i}^{-1}\left(L_{i}( \pm \infty)-\int_{t}^{\infty} C(s, t) d s\right)
$$

where $i$ is determined by $(-1)^{i} x(t)<0$ for $t \geq t_{0}$. In particular, if $l(x)=x^{\gamma}, \gamma$ $>1, \gamma$ the quotient of odd integers,

$$
|x(t)| \leq\left[(\gamma-1) \int_{t}^{\infty} C(s, t) d s\right]^{1 /(1-\gamma)} .
$$

Proof. Let $x(t)$ be a nonoscillatory solution of (1.1) which is eventually positive. It follows from Theorem 5(ii) that, for every $a$ sufficiently large,

$$
L_{1}(\infty)-L_{1}(x(a)) \geq \int_{a}^{\infty} C(s, a) d s
$$

or, equivalently,

$$
x(a) \leq L_{1}^{-1}\left(L_{1}(\infty)-\int_{a}^{\infty} C(s, a) d s\right)
$$

if $a$ is chosen large enough so that $L_{1}(\infty)-\int_{a}^{\infty} C(s, a) d s \geq 0$. If $x(t)$ is eventually negative, the argument is similar. As (3.14) is just a restatement of (3.13) in a special case, the proof is complete.

As an illustration of the estimate given by (3.14), consider the ordinary differential equation

$$
x^{\prime \prime}+\frac{1}{4} t^{-(m+2)} x^{2 m+1}=0,
$$

$m$ a positive integer, which was analyzed by Moore and Nehari [16] to illustrate the possible types of behavior of the solutions of the equation

$$
x^{\prime \prime}+c(t) x^{2 m+1}=0 .
$$

From the analysis given by Moore and Nehari for equation (3.15) it follows that every nonoscillatory solution of (3.15) satisfies $|x(t)| \leq(m+1)^{1 / 2 m} t^{1 / 2}$ while the estimate obtained from (3.14) is $|x(t)| \leq(2 m+2)^{1 / 2 m} t^{1 / 2}$.

A number of results relate the growth of nonoscillatory solutions to a power of t. As an easy corollary of (3.14) we present the following result which can easily be shown to include Theorem IX of [16] which gives a similar result for (3.16).

Corollary 2. Let $a(t)$ be positive and continuous for $t \geq 0$ and $\gamma>1$ be the quotient of odd integers. If $x(t)$ is an eventually positive nonoscillatory solution of

$$
\left.x^{(n)}(t)+a(t) x^{\gamma}(t)=0 \quad \text { ( } n \text { even }\right)
$$


and

$$
\liminf _{t \rightarrow \infty} t^{\beta(\gamma-1)} \int_{t}^{\infty} s^{n-1} a(s) d s>0
$$

for some positive constant $\beta$, then there is a constant $M>0$ so that, for all large $t$, $x(t) \leq M t^{\beta}$.

Proof. Here, $f(t, x)=a(t) x^{\gamma}$ and, thus, it follows from (3.14) that we need only show

$$
\int_{t}^{\infty}(s-t)^{n-1} a(s) d s \geq M_{1} t^{\beta(1-\gamma)}
$$

or

$$
t^{\beta(\gamma-1)} \int_{t}^{\infty}(s-t)^{n-1} a(s) d s \geq M_{1}>0
$$

for some constant $M_{1}$ for all large $t$ to obtain the result. For $s \geq 2 t$,

$$
t^{\beta(\gamma-1)} \int_{t}^{\infty}(s-t)^{n-1} a(s) d s \geq K(2 t)^{\beta(\gamma-1)} \int_{2 t}^{\infty} s^{n-1} a(s) d s
$$

where $K=2^{\beta(1-\gamma)+1-n}$, and the result follows.

We present next an example of an equation for which no result known to the author appears to apply but which is tractable using Theorem 5 . We consider equation

$$
x^{\prime \prime}+M_{1} t^{\alpha} x^{\beta}+M_{2} t^{\gamma} x^{\delta}=0
$$

where $M_{1}, M_{2}, \beta$ and $\delta$ are positive constants, $\beta$ and $\delta$ the quotients of odd integers satisfying $0<\beta<1<\delta$. Suppose, also, that $\alpha$ and $\gamma$ are constants satisfying $-\delta-1<\gamma<-2<\alpha<-\beta-1$. It follows from Theorem $A$ that both of the equations

$$
x^{\prime \prime}+M_{1} t^{\alpha} x^{\beta}=0 \text { and } x^{\prime \prime}+M_{2} t^{\gamma} x^{8}=0
$$

have nonoscillatory solutions.

Define $b(t)=M_{1} t^{\alpha}, c(t)=M_{2} t^{\gamma}, k(x)=x^{\beta}, l(x)=x^{\delta-\varepsilon}$, and $m(x)=x^{\varepsilon}$ where $\delta-1>\varepsilon>0$ and $\varepsilon=p / q$ where $p$ is an even integer and $q$ is an odd integer. It follows from Theorem $5(\mathrm{i})$ and an easy calculation that every solution of (3.18) is oscillatory if we can choose $\varepsilon$ with the property that $(\alpha+2) /(\beta-1)$ $\leq(\gamma+2) / \varepsilon$. As $\delta-1>\varepsilon>0$, we see that this choice can be made if and only if $(\alpha+2) /(\beta-1)<(\gamma+2) /(\delta-1)$. We note that this result is best possible for if $(\alpha+2) /(1-\beta)=(\gamma+2) /(1-\delta)=\mu$, then $t^{\mu}$ is a solution of (3.18) if $M_{1}$ and $M_{2}$ are chosen so that $M_{1}+M_{2}=-\mu(\mu-1)$.

In a similar manner, for the equation

$$
x^{\prime \prime}+M_{1} t^{-2} x+M_{2} t^{\gamma} x^{8}=0
$$


where $M_{2}, \gamma$, and $\delta$ are as above, and $0<M_{1} \leq \frac{1}{4}$, it follows from Theorem 5(i) and an easy calculation that all solutions of (3.19) are oscillatory if $M_{1}$ $>(\gamma+2) /(1-\delta)$.

From the remarks after Lemma 4 we see that rather than using equation (2.2), we may use the equation

$$
u^{\prime}=u^{2} f\left(t, d t^{n-1} / u\right)
$$

in conjunction with Lemmas 2 and 3 to obtain results for equation (1.1) in the sublinear case.

Theorem 6. Suppose $f$ satisfies

$$
x^{3} f\left(t, d t^{n-1} / x\right) \geq x[b(t) k(x)+c(t) l(x) m(x)]
$$

for all $x$ and $t \geq 0$, where $b, c, k, l$, and $m$ satisfy (3.4) and (3.5) with $\omega_{1}(t, r)=b(t) k(x)+c(t) l(x) m(x)$.

(i) Every solution of (1.1) is oscillatory if, for each $p \neq 0$ and $a \geq 0$,

$$
\int_{a}^{\infty} c(t) m\left(K_{i}^{-1}\left(K_{i}(p)+\int_{a}^{t} b(s) d s\right)\right) d t=\infty
$$

where $(-1)^{i} p<0$.

(ii) Suppose $\Psi_{1}(t)>0>\Psi_{2}(t)$ are functions which satisfy

$L_{i}( \pm \infty)-L_{i}\left(\Psi_{i}(a)\right)<\int_{a}^{\infty} c(t) m\left(K_{i}^{-1}\left(K_{i}\left(\Psi_{i}(a)\right)+\int_{a}^{t} b(s) d s\right)\right) d t, \quad i=1,2$.

If $x(t)$ is a nonoscillatory solution of $(1.1), x^{(n-1)}(t)$ satisfies $x^{(n-1)}(t)>1 / \Psi_{1}(t)$ or $x^{(n-1)}(t)<1 / \Psi_{2}(t)$ for all large $t$ according as to whether $x(t)$ is eventually positive or eventually negative.

Proof. The first statement follows from Theorem 3(ii) and Lemma 2 applied to (3.20) and the fact that every solution of (3.20) has finite escape time if and only if every solution of (2.2) becomes zero in finite time. The second statement follows from Lemma 3 and Theorem 2 as solutions of (2.2) with initial condition $v\left(t_{0}\right)$ satisfying $1 / \Psi_{1}\left(t_{0}\right) \geq v\left(t_{0}\right) \geq 1 / \Psi_{2}\left(t_{0}\right)$ must become zero in finite time.

If $f(t, x)=c(t) x^{\gamma}, \gamma$ the quotient of odd integers, $0<\gamma<1$, and $c(t)$ is positive and continuous for $t \geq 0$, we see that Theorem 6(i) is a restatement of the sufficiency criterion of the second part of Theorem A.

While it is possible to obtain growth estimates for nonoscillatory solutions of (1.1) using Theorem 6(ii), it is frequently more convenient to use the estimate from equation (2.1a) and Theorem 1.

Theorem 7. Suppose $x f(t, x) \geq c(t) x^{\gamma+1}$ where $c(t)$ is positive and continuous for $t \geq 0$ and $\gamma$ is the quotient of odd integers with $0<\gamma<1$. If $x(t)$ is a nonoscillatory solution of (1.1), 


$$
|x(t)| \geq\left[|x(a)|^{1-\gamma}+(1-\gamma) \int_{a}^{t} C(s, a) d s\right]^{1 /(1-\gamma)}
$$

for $t \geq$ a a sufficiently large. If

$$
\liminf _{t \rightarrow \infty} t^{\beta(\lambda-1)} \int_{a}^{t} C(s, a) d s>0
$$

for some $\beta>0$, then, for all large $t, x(t)$ satisfies $|x(t)| \geq M t^{\beta}$ for some positive constant $M$. The function $C$ is given by $C(t, a)=(t-a)^{n-1} c(t) /(n-1)$ !.

Proof. The estimate (3.21) is obtained by solving the equation $u^{\prime}=C(t, a) u^{\gamma}$ and applying Theorem 1. The second statement follows from (3.21) by an argument similar to that used in Corollary 2.

If we consider the equation

$$
x^{(n)}+t^{-0} x^{\gamma}=0
$$

where $\gamma$ is the quotient of odd integers, $0<\gamma<1$, and $\sigma$ is constant with $(n-1) \gamma+1<\sigma<n$, it is easy to see that $c t^{\beta}$ is a solution of (3.23) if $c$ is an appropriately chosen constant and $\beta=(\sigma-n) /(\gamma-1)$. Also, an easy calculation shows that (3.22) is satisfied with $\beta=(\sigma-n) /(\gamma-1)$.

4. We now consider the delay-differential equation

$$
x^{(n)}(t)+h\left(t, x(t), x\left(q_{1}(t)\right), \ldots, x\left(q_{m}(t)\right)\right)=0 \quad(n \text { even })
$$

where $h\left(t, x_{1}, \ldots, x_{m+1}\right)$ is continuous on $[0, \infty) \times R^{m+1}$ into $R$, nondecreasing in $x_{1}, \ldots, x_{m+1}$ for fixed $t$, and if $x_{1}, \ldots, x_{m+1}$ have the same sign then $h\left(t, x_{1}, \ldots\right.$, $\left.x_{m+1}\right)$ has that sign. Also, we assume $q_{i}$ is continuous for $t \geq 0, q_{i}(t) \leq t$, and $q_{i}(t) \rightarrow \infty$ as $t \rightarrow \infty, i=1, \ldots, m$.

Unfortunately, under the above assumptions it is possible for nonoscillatory solutions of (4.1) to have finite escape time. This fact is easily illustrated by examining the equation

$$
x^{\prime \prime}(t)+x(q(t)) x^{2}(t)=0
$$

with an appropriate $q(t)$ and initial function so that on a sufficiently large interval the solution of (4.2) under consideration is actually a positive solution of $x^{\prime \prime}(t)-x^{2}(t)=0$.

There are, however, a number of reasonable conditions which will guarantee that nonoscillatory solutions of (4.1) must exist on a positive half-line. For example, using the techniques of [1], it can be shown that if (4.1) is of the form

$$
x^{(n)}(t)+h(t, x(q(t)))=0
$$

or if $q_{i}(t)<t, i=1, \ldots, m$, and there exists a continuous function $g$ so that

$$
h\left(t, x_{1}, x_{2}, \ldots, x_{m}\right) \geq(\leq) g\left(t, x_{2}, \ldots, x_{m}\right)
$$


when $x_{1} \geq(\leq) 0$, nonoscillatory solutions of (4.1) must exist on a positive halfline. There are a number of other criteria which ensure the same conclusion and we shall assume without further remark that nonoscillatory solutions of (4.1) do not have finite escape time.

The next lemma is an extension of a lemma obtained by Erbe [4] when considering the oscillation problem for second-order delay-differential equations.

Lemma 4. Let $y(t)$ be a function such that it and all its derivatives up to order $m$ inclusive exist and are of constant sign in the interval $\left(t_{0}, \infty\right)$ with $y^{(m)}(t) y(t) \leq 0$. Let $q(t)$ be a continuous function defined for $t \geq t_{0}$ with the property that $q(t) \leq t$ and $q(t) \rightarrow \infty$ as $t \rightarrow \infty$. If, for $t \geq t_{1} \geq t_{0}, y^{(k)}(q(t))>0, k=0,1, \ldots, l$, while $y^{(l+1)}(q(t))<0$ where $l>0$ is the integer specified in Lemma 1, then, for any constant $\alpha, 0<\alpha<1$, there exists $T$ so that, for $t \geq T$,

$$
t^{m-1} y(q(t)) \geq \alpha q^{m-1}(t) y(t) .
$$

Proof. If $q(t)=t$ there is nothing to prove and we assume that, at the value of $t$ under consideration, $q(t)<t$. From the Taylor expansion of $y(t)$ and the fact that $y^{(t+1)}<0$, we obtain, for $t \geq t_{1}$,

$$
y(t) \leq \sum_{j=0}^{l} y^{(j)}(q(t))(t-q(t))^{j} /(j !) .
$$

Dividing by $y(q(t))$ and applying Lemma 1(iv) repeatedly, we see that

$$
\begin{aligned}
y(t) / y(q(t)) & \leq \sum_{j=0}^{l} C(l, j)\left[(t-q(t)) /\left(q(t)-t_{0}\right)\right]^{j} \\
& =\left[\left(t-t_{0}\right) /\left(q(t)-t_{0}\right)\right]^{l}
\end{aligned}
$$

for sufficiently large $t$. The result now follows easily from this inequality as $l<m, q(t) \leq t$ and $q(t) \rightarrow \infty$ as $t \rightarrow \infty$.

Consider now the first-order scalar equations

$$
u^{\prime}=(t-a)^{n-1} h\left(t, u, \mu_{1}(t) u, \ldots, \mu_{m}(t) u\right) /(n-1) !
$$

and

$$
v^{\prime}=-h\left(t, d t^{n-1} v, \alpha d q_{1}^{n-1}(t) v, \ldots, \alpha d q_{m}^{n-1}(t) v\right)
$$

where $d=\left(\frac{1}{2}\right)^{n(n-1)}(1 /(n-1) !), \alpha$ is a fixed but arbitrary constant satisfying $0<\alpha<1$ and $\mu_{i}(t)=\alpha\left(q_{i}(t) / t\right)^{n-1}, i=1, \ldots, m$. It is clear that with the aid of Lemma 4, a theorem corresponding to Theorem 1 relating the nonoscillatory solutions of (4.1) to the solutions of (4.3a) and (4.4) can be proven with only minor modifications in the proof. In a similar fashion, all that follows from Theorem 1 requires at most a minor modification in either the proof or the statement of the theorem. We leave these modifications to the interested reader. 


\section{REFERENCES}

1. T. Burton and R. Grimmer, Oscillation, continuation, and uniqueness of solutions of retarded differential equations, Trans. Amer. Math. Soc. 179 (1973), 193-209.

2.- Oscillatory solutions of $x^{\prime \prime}(t)+a(t) f(x(q(t)))=0$, Delay and Functional Differential Equations and Their Applications, Academic Press, New York, 1972, pp. 335-343.

3. C. V. Coffman and D. F. Ulrich, On the continuation of solutions of a certain nonlinear differential equation, Monatsh. Math. 71 (1967), 385-392. MR 37 \#3078.

4. L. Erbe, Oscillations in functional differential equations, Japan-United States Seminar on Ordinary Differential and Functional Equations, Lecture Notes in Math., Vol. 243, Springer-Verlag, New York, 1971, pp. 289-292.

5. H. Gollwitzer, On nonlinear oscillation for a second order delay equation, J. Math. Anal. Appl. 26 (1969), 385-389. MR 39 \#581.

6. R. Grimmer, Stability of a scalar differential equation, Proc. Amer. Math. Soc. 32 (1972), 452-456. MR 44 \#5567.

7. T. G. Hallam and J. W. Heidel, Structure of the solution set of some first order differential equations of comparison type, Trans. Amer. Math. Soc. 160 (1971), 501-512. MR 43 \#7709.

8. S. P. Hastings, Boundary value problems in one differential equation with a discontinuity, J. Differential Equations 1 (1965), 346-369. MR 31 \#4954.

9. A. Kartsatos, On oscillation of solutions of even order nonlinear differential equations, J. Differential Equations 6 (1969), 232-237. MR 39 \#5877.

10. I. T. Kiguradze, Oscillation properties of solutions of certain ordinary differential equations, Dokl. Akad. Nauk SSSR 144 (1962), 33-36 = Soviet Math. Dokl. 3 (1962), 649-652. MR 25 \#278.

11.—, The problem of oscillation of solutions of nonlinear differential equations, Differencial'nye Uravnenija 8 (1965), 995-1006 = Differential Equations 8 (1965), 773-782. MR 33 \#2896.

12. G. Ladas, Oscillation and asymptotic behavior of solutions of differential equations with retarded argument, J. Differential Equations 10 (1971), 281-290. MR 45 \#681.

13. V. Lakshmikantham and S. Leela, Differential and integral inequalities, vol. 1, Academic Press, New York, 1969.

14. I. Ličko and M. Švec, Le caractère oscillatoire des solutions de l'équation $y^{(n)}+f(x) y^{\alpha}=0, n$ $>1$, Czechoslovak Math. J. (88) 13 (1963), 481-491.MR 28 \#4210.

15. J. W. Macki and J. S. W. Wong, Oscillation of solutions to second order nonlinear differential equations, Pacific J. Math. 24 (1968), 111-117. MR 37 \# 507.

18. R. A. Moore and Z. Nehari, Nonoscillation theorems for a class of nonlinear differential equations, Trans. Amer. Math. Soc. 93 (1959), 30-52. MR 22 \#2755.

17. H. Onose, Oscillatory property of ordinary differential equations of arbitrary order, J. Differential Equations 7 (1970), 454-458. MR 41 \#2116.

18. G. H. Ryder and D. V. V. Wend, Oscillation of solutions of certain ordinary differential equations of nth order, Proc. Amer. Math. Soc. 25 (1970), 463-469. MR 41 \#5710.

19. J. S. W. Wong, Second order oscillation with retarded arguments, Ordinary Differential Equations 1971 NRL-MRC Conference, Academic Press, New York, 1972, pp. 581-596.

Department of Mathematics, Southern Illinois University, Carbondale, Illinois 62901 